\title{
TWISTED ALEXANDER POLYNOMIALS, SYMPLECTIC 4-MANIFOLDS AND SURFACES OF MINIMAL COMPLEXITY
}

\author{
STEFAN FRIEDL \\ Université du Québec à Montréal, Montréal, Québec, Canada \\ and University of Warwick, Coventry, UK \\ E-mail:sfriedl@gmail.com \\ STEFANO VIDUSSI \\ Department of Mathematics, University of California \\ Riverside, CA 92521, USA \\ E-mail:svidussi@math.ucr.edu
}

\begin{abstract}
Let $M$ be a 4-manifold which admits a free circle action. We use twisted Alexander polynomials to study the existence of symplectic structures and the minimal complexity of surfaces in $M$. The results on the existence of symplectic structures summarize previous results of the authors in [FV08a, FV08b, FV07]. The results on surfaces of minimal complexity are new.
\end{abstract}

\section{Introduction and main results}

1.1. 4-manifolds with free circle action. Let $M$ be a 4-manifold which admits a free circle action. (Throughout the paper, unless otherwise stated, we will assume that all manifolds are closed, oriented and connected.) In this paper we will discuss how twisted Alexander polynomials give information, for this class of manifolds, on two central problems in 4-dimensional topology, namely the study of the existence of symplectic structures, and the minimal complexity of surfaces in $M$.

We start by recalling some simple facts about this class of manifolds, as they will be frequently used in what follows. The existence of a free $S^{1}$-action on $M$ implies that $M$ is a principal $S^{1}$-bundle, so that there is a projection map $p: M \rightarrow N$ where we denote by $N$ the orbit space of the free circle action. This principal bundle is determined

2000 Mathematics Subject Classification: 57R17, 57M27.

Key words and phrases: twisted Alexander polynomial, symplectic manifold, circle action, Thurston norm.

The paper is in final form and no version of it will be published elsewhere. 
by its Euler class $e \in H^{2}(N ; \mathbb{Z})$. Note that $e=0$ if and only if $M$ is a product bundle $M=S^{1} \times N$. We have the Gysin sequence

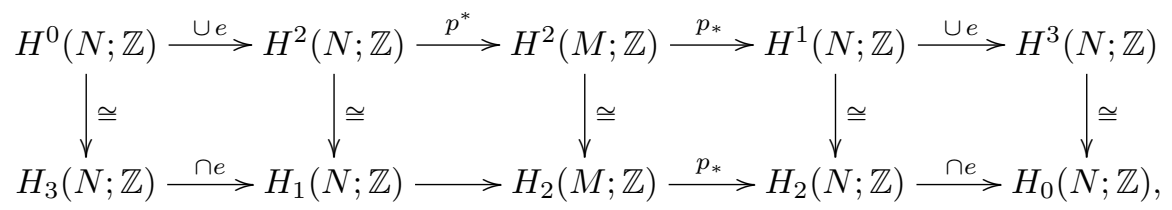

where $p_{*}: H^{2}(M ; \mathbb{Z}) \rightarrow H^{1}(N ; \mathbb{Z})$ denotes integration along the fiber. From this sequence we can immediately deduce that

$$
b_{2}(M)=\left\{\begin{aligned}
2 b_{1}(N), & \text { if } e \text { is torsion, } \\
2 b_{1}(N)-2, & \text { if } e \text { is non-torsion. }
\end{aligned}\right.
$$

One can easily see that the intersection form vanishes on the half-dimensional space $\operatorname{Im}\left\{H_{1}(N ; \mathbb{Q}) \rightarrow H_{2}(M ; \mathbb{Q})\right\} \subset H_{2}(M ; \mathbb{Q})$. It follows that $b_{2}^{+}(M)=b_{2}^{-}(M)$, so that $\operatorname{sign}(M)=0$. For sake of simplicity, we will restrict ourselves in this paper to the case that $e$ is either trivial or non-torsion. Also, we will assume that $b_{2}^{+}(M)>1$, but the techniques and results presented here extend to the torsion case and, with the usual caveats, to the case of $b_{2}^{+}(M)=1$. We refer to [FV08a, FV08b, FV07] for the details of these cases. Finally, note that the long exact homotopy sequence of the fibration shows that the $\operatorname{map} p_{*}: \pi_{1}(M) \rightarrow \pi_{1}(N)$ is an epimorphism.

1.2. Symplectic 4-manifolds and fibered 3-manifolds. Remember that a 4-manifold is called symplectic if it admits a closed, non-degnerate 2 -form $\omega$. Our first goal is to study the question of which 4-manifolds with free circle actions are symplectic. Let $M$ be a 4-manifold as above. It is well-known that if $(N, \phi)$ fibers over $S^{1}$ for some $\phi \in H^{1}(N, \mathbb{Z})$ with $\phi \cup e=0$, then $M$ admits a symplectic structure. We refer to [Th76], [Bou88], [FGM91] and [FV07] for details. (Here we say that $(N, \phi)$ fibers over $S^{1}$ if the homotopy class of maps $N \rightarrow S^{1}$ determined by $\phi \in H^{1}(N ; \mathbb{Z})=\left[N, S^{1}\right]$ contains a representative that is a fiber bundle over $S^{1}$.) It is natural to ask whether the converse of this statement holds true. We point out that the openness of the symplectic condition implies that if $M$ is symplectic then $M$ also has a symplectic form representing an integral cohomology class. We will implicitly make such a choice whenever necessary. Finally, if $\omega$ is a symplectic form it follows immediately from $[\omega]^{2} \neq 0 \in H^{4}(M ; \mathbb{R})$ that $p_{*}[\omega] \neq 0 \in H^{1}(N ; \mathbb{R})$.

The problem of studying the existence of symplectic forms on $M$ can be summarized in terms of the following conjecture:

Conjecture 1.1. Let $M$ be a 4-manifold with a free circle action with orbit space $N$. If $M$ admits a symplectic structure $\omega$ with $[\omega] \in H^{2}(N ; \mathbb{Z})$, then $\left(N, p_{*}[\omega]\right)$ fibers over $S^{1}$.

This problem was first raised in [FGM91], but only since the appearance of Taubes' results on Seiberg-Witten invariants of symplectic 4-manifolds (see [Ta94, Ta95]) are there tools at hand to seriously tackle the conjecture. We refer to [Kr98], [Kr99], [McC01], [Ba01], [Vi03] for results supporting this conjecture. 
1.3. Surfaces of minimal complexity. Given a surface $\Sigma$ with connected components $\Sigma_{1}, \ldots, \Sigma_{k}$ we define its complexity to be

$$
\chi^{-}(\Sigma)=\sum_{i=1}^{k} \max \left\{-\chi\left(\Sigma_{i}\right), 0\right\}
$$

We say that an embedded surface $\Sigma$ in a (3- or 4-) manifold has minimal complexity if it minimizes the complexity in its homology class.

Given a 3-manifold $N$ and $\phi \in H^{1}(N ; \mathbb{Z})$ the Thurston norm on $H^{1}(N, \mathbb{Z})$ is defined as

$$
\chi_{N}^{-}(\phi)=\chi^{-}(\Sigma)
$$

where $\Sigma$ is a surface of minimal complexity dual to $\phi$. Given a 4-manifold $M$ and $\sigma \in$ $H^{2}(M ; \mathbb{Z})$ we also define

$$
\chi_{M}^{-}(\sigma)=\chi^{-}(\Sigma)
$$

where $\Sigma$ is a surface of minimal complexity dual to $\phi$. (Note that in spite of the similar definition, the minimal complexity function on 4-manifolds is not known to share the properties of the Thurston norm, e.g. linearity. We refer to [Kr98, Section 7] for more related open questions.)

Let $M$ be a 4-manifold with $b_{2}^{+}(M)>1$ and consider a class $\sigma \in H^{2}(M ; \mathbb{Z})$; then the adjunction inequality says that

$$
\chi_{M}^{-}(\sigma) \geq \sigma \cdot \sigma+\sigma \cdot \kappa
$$

for any Seiberg-Witten basic class $\kappa \in H^{2}(M ; \mathbb{Z})$. Even though this inequality gives useful lower bounds, it is in general not enough to determine the function $\chi_{M}^{-}: H^{2}(M ; \mathbb{Z}) \rightarrow \mathbb{N}$. In this paper we are interested in the study of this function for 4-manifolds $M$ with a free circle action. Note that it is not difficult to see, in this case (cf. Section 3.1), that we can put the absolute value to $\sigma \cdot \sigma$ in equation (3).

Given $\sigma \in H^{2}(M ; \mathbb{Z})$ we say that $\sigma$ has property $(*)$ if there exists a (possibly disconnected) embedded surface $\Sigma \subset N$ and a (possibly disconnected) closed curve $c \subset N$ in general position with the following properties:

1. $\Sigma$ is a Thurston norm minimizing surface dual to $p_{*}(\sigma)$,

2. Given a lift $\tilde{\Sigma}$ of $\Sigma$ to $M$ (which by Lemma 2.1 always exists) the singular surface $p^{-1}(c) \cup \tilde{\Sigma}$ represents $P D(\sigma)$,

3. The geometric intersection number of $\Sigma$ and $c$ is given by the absolute value of the algebraic intersection number $\Sigma \cdot c$.

Property $(*)$ is the suitable generalization of the property defined by Kronheimer in [Kr99] for the product case. We will see in Section 2.1 that property $(*)$ is fairly common; in particular, it is satisfied whenever the Alexander polynomial $\Delta_{N, p_{*}(\sigma)} \neq 0 \in \mathbb{Z}\left[t^{ \pm 1}\right]$. On the other hand we will also see that, for suitable $N$, there exist $\sigma$ which do not satisfy (*). In Section 2.1 we prove the following lemma (cf. also [Kr99, Section 1.2]).

Lemma 1.2. Let $\sigma \in H^{2}(M ; \mathbb{Z})$ that satisfies $(*)$. Then

$$
\chi_{M}^{-}(\sigma) \leq \chi_{N}^{-}\left(p_{*}(\sigma)\right)+|\sigma \cdot \sigma| .
$$


Kronheimer [Kr99, Corollary 2] [Kr98, Corollary 7.6] proved that in the case that $M$ is a product $S^{1} \times N$, in many cases this is in fact an equality. More precisely, Kronheimer proved the following result.

THEOREM 1.3. Let $N$ be an irreducible 3-manifold whose Thurston norm is not totally degenerate, $M=S^{1} \times N$ and $\sigma \in H^{2}\left(S^{1} \times N ; \mathbb{Z}\right)$. Then

$$
\chi_{M}^{-}(\sigma) \geq \chi_{N}^{-}\left(p_{*}(\sigma)\right)+|\sigma \cdot \sigma| \text {. }
$$

Note that Kronheimer showed that it is not always necessary to assume that $N$ is irreducible (cf. [Kr99, Theorem 10]) and to assume that the Thurston norm is nondegenerate (cf. [Kr99, Section 1.2]). It now seems reasonable to pose the following question (cf. also [Kr98, Question 7.12]).

Question 1.4. Let $M \rightarrow N$ be a principal $S^{1}$-bundle. Given $\sigma \in H^{2}(M ; \mathbb{Z})$, does the inequality

$$
\chi_{M}^{-}(\sigma) \geq \chi_{N}^{-}\left(p_{*}(\sigma)\right)+|\sigma \cdot \sigma|
$$

always hold? Is this an equality even for $\sigma$ which do not satisfy $(*)$ ?

Note that given $\sigma \in H_{2}(M ; \mathbb{Z})$ we always have the inequality

$$
\chi_{M}^{-}(\sigma) \geq \chi_{N}^{-}\left(p_{*}(\sigma)\right) .
$$

This follows from Gabai's result [Ga83] that for any singular surface $S^{\prime} \subset N$ dual to some $\phi \in H^{1}(N ; \mathbb{Z})$ we have $\chi^{-}\left(S^{\prime}\right) \geq \chi_{N}^{-}(\phi)$.

1.4. Statement of the main results. Given a 3 -manifold $N, \phi \in H^{1}(N ; \mathbb{Z})$ and an epimorphism $\alpha: \pi_{1}(N) \rightarrow G$ onto a finite group we can define the 1-variable twisted Alexander polynomial $\Delta_{N, \phi}^{\alpha} \in \mathbb{Z}\left[t^{ \pm 1}\right]$. We refer to Section 2.2 for details. Given a non-zero Laurent polynomial $\sum_{i=n}^{m} a_{i} t^{i}$ with $a_{n} \neq 0$ and $a_{m} \neq 0$ we define

$$
\operatorname{deg}\left(\sum_{i=n}^{m} a_{i} t^{i}\right)=m-n ;
$$

furthermore we define $\operatorname{deg}(0)=-\infty$. Given an integer $n$ we write $-\infty+n=-\infty$ and $-\infty<n$.

We will show that the degrees of twisted Alexander polynomials give lower bounds on $\chi_{M}^{-}(\sigma)$ for a given $\sigma \in H^{2}(M ; \mathbb{Z})$. Note that if $p_{*}(\sigma)=0 \in H^{1}(N ; \mathbb{Z})$, then $\sigma$ is clearly dual to a union of embedded tori and hence $\chi_{M}^{-}(\sigma)=0$. We therefore restrict ourselves now to the case that $\phi=p_{*}(\sigma) \neq 0 \in H^{1}(N ; \mathbb{Z})$.

Given $\phi \in H^{1}(N, \mathbb{Z})=\operatorname{Hom}\left(\pi_{1}(N), \mathbb{Z}\right)$ and a homomorphism $\alpha: \pi_{1}(N) \rightarrow G$, we denote by $\phi_{\alpha}$ its restriction to $\operatorname{ker} \alpha \subset \pi_{1}(N)$. Furthermore we denote by div $\phi_{\alpha}$ the divisibility of $\phi_{\alpha}$, i.e. the largest integer $n$ such that $\frac{1}{n} \phi_{\alpha}$ still defines an integral class. Our main theorem is now the following.

THEOREM 1.5. Let $M$ be a 4-manifold admitting a free circle action such that $b_{2}^{+}(M)>1$ and such that either $e=0$ or $e$ is non-torsion. Let $\sigma \in H^{2}(M ; \mathbb{Z})$ such that $\phi=p_{*}(\sigma) \neq$ $0 \in H^{1}(N ; \mathbb{Z})$. Then for any epimorphism $\alpha: \pi_{1}(N) \rightarrow G$ onto a finite group we have

$$
\chi_{M}^{-}(\sigma) \geq \frac{1}{|G|}\left(\operatorname{deg}\left(\Delta_{N, \phi}^{\alpha}\right)-2 \operatorname{div} \phi_{\alpha}\right)+|\sigma \cdot \sigma| .
$$

Furthermore, if $\sigma$ is represented by a symplectic form, then $\Delta_{N, \phi}^{\alpha}$ is monic. 
Note that the last statement is already contained in [FV08a] and [FV07]. The proof of the theorem relies on the adjunction inequality and Taubes' results applied to finite coverings of $M$. The resulting information on Seiberg-Witten invariants can be translated into information on twisted Alexander polynomials using the results of Meng and Taubes [MT96], Baldridge [Ba01], [Ba03] and Shapiro's lemma.

This theorem allows us to study the existence of symplectic structures and the complexity of surfaces using twisted Alexander polynomials. In [FV08a], [FV08b] and [FV07] the authors showed that twisted Alexander polynomials are very efficient at detecting fibered 3-manifolds (cf. also [Ch03], [GKM05], [FK06] and [Ki08]). The main result therein contained is the following.

THEOREM 1.6. Let $N$ be a 3-manifold. Let $\phi \in H^{1}(N ; \mathbb{Z})$ be non-trivial. If $\Delta_{N, \phi}^{\alpha} \neq 0$ for all epimorphisms $\alpha: \pi_{1}(N) \rightarrow G$ onto finite groups, then $N$ is prime. Furthermore, if $N$ has either vanishing Thurston norm or is a graph manifold, then $(N, \phi)$ fibers over $S^{1}$.

The primeness conclusion has first been proved by McCarthy [McC01]. In particular the combination of Theorems 1.5 and 1.6 gives an affirmative answer to Conjecture 1.1 if the orbit space $N$ has vanishing Thurston norm or if $N$ is a graph manifold. (For the case of vanishing Thurston norm, a proof of the Conjecture along the same lines is presented in [Bow07].)

Whereas twisted Alexander polynomials are good at detecting fibered 3-manifolds, their record at detecting the Thurston norm of a given $\phi \in H^{1}(N ; \mathbb{Z})$ is rather mixed. On the one hand we prove in Section 4 the following result.

THEOREM 1.7. There exists a 4-manifold $M$ with free circle action with non-torsion Euler class and $\sigma \in H^{2}(M ; \mathbb{Z})$ where the adjunction inequality is not strong enough to determine $\chi_{M}^{-}(\sigma)$, but where the bounds from Theorem 1.5 on $\chi_{M}^{-}(\sigma)$ coming from $\Delta_{N}^{\alpha}$ for an appropriate $\alpha: \pi_{1}(N) \rightarrow G$ determine $\chi_{M}^{-}(\sigma)$.

On the other hand we will see in Lemma 4.2 that if the ordinary Alexander polynomial $\Delta_{N, \phi}$ vanishes, then all twisted Alexander polynomials $\Delta_{N, \phi}^{\alpha}$ vanish as well. Note that this effect would not happen if we used twisted Alexander polynomials corresponding to general representations $\alpha: \pi_{1}(N) \rightarrow \mathrm{GL}(\mathbb{C}, k)$ as in [FK06]. Unfortunately these more general twisted Alexander polynomials seem to have no interpretation in terms of Seiberg-Witten invariants of covers.

\section{Preliminaries}

2.1. Surfaces and principal $S^{1}$-bundles. We first prove the following lemma regarding surfaces in $N$ and $M$.

Lemma 2.1. Let $M \rightarrow N$ be a principal $S^{1}$-bundle with Euler class e. Let $\sigma \in H^{2}(M ; \mathbb{Z})$ and $\Sigma \subset N$ an embedded surface dual to $p_{*}(\sigma)$. Then $\Sigma$ lifts to a surface in $M$.

Proof. Clearly it is enough to show that the principal $S^{1}$-bundle over $N$ restricted to $\Sigma$ is trivial. This in turn is equivalent to showing that $\left.e\right|_{\Sigma}=0 \in H^{2}(\Sigma ; \mathbb{Z})$. But since $\Sigma$ is dual to $p_{*}(\sigma)$, this is equivalent to the condition that $p_{*}(\sigma) \cup e=0$, which in turn is satisfied by the Gysin sequence (1). 
We have the following lemma (cf. also [Kr99]).

Lemma 2.2. Let $\sigma \in H^{2}(M ; \mathbb{Z})$ that satisfies $(*)$. Then

$$
\chi_{M}^{-}(\sigma) \leq \chi_{N}^{-}\left(p_{*}(\sigma)\right)+|\sigma \cdot \sigma| .
$$

Proof. Let $\Sigma, \tilde{\Sigma}$ and $c$ as in the definition of property $(*)$. At each singular point of $p^{-1}(c) \cup \tilde{\Sigma}$ we can replace a pair of transverse disks with an embedded annulus having the same oriented boundary. Note that each replacement increases the Euler number by 2. We therefore obtain a smooth surface $T$ representing the class dual to $\sigma$ with

$$
\chi^{-}(T)=\chi^{-}(\tilde{\Sigma})+\chi^{-}\left(p^{-1}(c)\right)+2|\Sigma \cdot c| .
$$

Note that $p^{-1}(c)$ is a union of tori, hence $\chi^{-}\left(p^{-1}(c)\right)=0$. As $2 \Sigma \cdot c=\sigma \cdot \sigma$, we get

$$
\chi^{-}(T)=\chi_{N}^{-}\left(p_{*}(\sigma)\right)+|\sigma \cdot \sigma| \text {. }
$$

We have the following criterion for $\sigma \in H^{2}(M ; \mathbb{Z})$ having property $(*)$.

Lemma 2.3. Let $\sigma \in H^{2}(M ; \mathbb{Z})$ and let $n=\operatorname{div}\left(p_{*}(\sigma)\right)$ be the divisibility of $p_{*}(\sigma) \in$ $H^{1}(N ; \mathbb{Z})$. If there exists a connected Thurston norm minimizing surface dual to the primitive class $\frac{1}{n} p_{*}(\sigma)$, then $\sigma$ has property $(*)$.

Proof. Let $\sigma \in H^{2}(M ; \mathbb{Z})$ and write $n=\operatorname{div}\left(p_{*}(\sigma)\right)$. Assume $\frac{1}{n} p_{*}(\sigma)$ is dual to a connected Thurston norm minimizing surface $\Sigma^{\prime}$. By [Th86] the union of $n$ parallel copies of $\Sigma^{\prime}$ is then a Thurston norm minimizing surface dual to $p_{*}(\sigma)$. By Lemma 2.1 we can lift $\Sigma^{\prime}$ to a surface $\tilde{\Sigma}^{\prime} \subset M$. It follows from the Gysin sequence (1) that we can find an embedded curve $c \in N$ such that the class dual to $\sigma$ is given by $\left[n \tilde{\Sigma}^{\prime}\right]+\left[p^{-1}(c)\right]$. We can assume that $c$ is in general position with $n \Sigma^{\prime}$, hence $n \tilde{\Sigma}^{\prime}$ and $p^{-1}(c)$ are in general position. Since $\Sigma^{\prime}$ is connected we can choose $c$ such that the geometric intersection number of $\Sigma^{\prime}$ and $c$ is given by the absolute value of $\Sigma^{\prime} \cdot c$. (Note that $c$ could be disconnected.)

The following lemma follows easily from [McM02, Proposition 6.1] or [Tu02].

Lemma 2.4. Let $\phi \in H^{1}(N ; \mathbb{Z})$ and $n=\operatorname{div}(\phi)$. If $\Delta_{N, \phi} \neq 0$, then there exists a connected Thurston norm minimizing surface dual to the primitive class $\frac{1}{n} \phi$.

REMARK. We now give examples of manifolds of the form $M=S^{1} \times N$ such that not every $\sigma \in H^{2}(M ; \mathbb{Z})$ has property $(*)$. For example, let $N$ be the connected sum of the zero framed surgeries on two non-trivial oriented knots $K_{1}$ and $K_{2}$. Let $\mu_{1}, \mu_{2} \in$ $H^{1}(N ; \mathbb{Z})$ be a basis given by the meridians of $K_{1}$ and $K_{2}$. Furthermore let $F_{1}, F_{2}$ be the result of capping off two minimal genus Seifert surfaces of $K_{1}$ and $K_{2}$. Consider $\phi=P D\left(a_{1}\left[F_{1}\right]\right)+P D\left(a_{2}\left[F_{2}\right]\right), a_{1}, a_{2} \in \mathbb{Z}$, then for $a_{1} \neq 0$ and $a_{2} \neq 0$ there exists no connected Thurston norm minimizing surface dual to $\phi$.

We now specialize to $a_{1}=3$ and $a_{2}=2$. Also, let $\gamma=\mu_{1}-\mu_{2}$. Then $\gamma \cdot \phi=1$. But it is easy to see that there exists no Thurston norm minimizing surface $\Sigma$ dual to $\phi$ and a curve $c$ representing $\gamma$ such that the geometric intersection number of $\Sigma$ and $c$ equals 1 . Now let $\sigma \in H^{2}\left(S^{1} \times N ; \mathbb{Z}\right)$ be the element which corresponds to $P D(\gamma)+\phi \otimes 1$ under the Künneth decomposition $H^{2}\left(S^{1} \times N ; \mathbb{Z}\right)=H^{2}(N ; \mathbb{Z}) \oplus H^{1}(N ; \mathbb{Z}) \otimes H^{1}\left(S^{1} ; \mathbb{Z}\right)$. Then it is clear that $\sigma$ does not have property $(*)$. 
Finally, let $\gamma=\mu_{1}+\mu_{2}$. Then $\gamma \cdot \phi=5$ and we can find a Thurston norm minimizing surface $\Sigma$ dual to $\phi$ and a curve $c$ representing $\gamma$ such that the geometric intersection number of $\Sigma$ and $c$ equals 5 . The corresponding element $\sigma \in H^{2}\left(S^{1} \times N ; \mathbb{Z}\right)$ has property (*), but does not satisfy the conditions of Lemma 2.3.

These examples show that in general it is not clear when $(*)$ is satisfied. Also, there does not seem to be a good conjecture for what $\chi_{M}^{-}(\sigma)$ should be in the case that $(*)$ does not hold.

2.2. Twisted Alexander polynomials. In this section we are going to recall the definition of the (twisted) Alexander polynomial associated to an epimorphism of the fundamental group of a compact 3-manifold onto a finite group. Twisted Alexander polynomials were first introduced for the case of knots by Xiao-Song Lin [Li01] and Lin's definition was later generalized to 3-manifolds by Wada [Wa94], Kirk-Livingston [KL99] and Cha [Ch03].

Let $N$ be a compact 3-manifold, $\phi \in H^{1}(N ; \mathbb{Z})=\operatorname{Hom}\left(\pi_{1}(N), \mathbb{Z}\right)$ and let $\alpha: \pi_{1}(N) \rightarrow$ $G$ be an epimorphism onto a finite group $G$. Then $\alpha \times \phi$ gives an action of $\pi_{1}(N)$ on $G \times \mathbb{Z}$, which extends to a ring homomorphism from $\mathbb{Z}\left[\pi_{1}(N)\right]$ to the $\mathbb{Z}\left[t^{ \pm 1}\right]$-linear endomorphisms of $\mathbb{Z}[G \times \mathbb{Z}]=\mathbb{Z}[G]\left[t^{ \pm 1}\right]$. This induces a left $\mathbb{Z}\left[\pi_{1}(N)\right]$-structure on $\mathbb{Z}[G]\left[t^{ \pm 1}\right]$.

Now let $\tilde{N}$ be the universal cover of $N$. Note that $\pi_{1}(N)$ acts on the left on $\tilde{N}$ as group of deck transformation. The chain groups $C_{*}(\tilde{N})$ are in a natural way right $\mathbb{Z}\left[\pi_{1}(N)\right]$ modules, with the right action on $C_{*}(\tilde{N})$ defined via $\sigma \cdot g:=g^{-1} \sigma$, for $\sigma \in C_{*}(\tilde{N})$. We can form by tensor product the chain complex $C_{*}(\tilde{N}) \otimes_{\mathbb{Z}\left[\pi_{1}(N)\right]} \mathbb{Z}[G]\left[t^{ \pm 1}\right]$. Now define $H_{i}\left(N ; \mathbb{Z}[G]\left[t^{ \pm 1}\right]\right):=H_{i}\left(C_{*}(\tilde{N}) \otimes_{\mathbb{Z}\left[\pi_{1}(N)\right]} \mathbb{Z}[G]\left[t^{ \pm 1}\right]\right)$, which inherit the structure of $\mathbb{Z}\left[t^{ \pm 1}\right]-$ modules. These modules are called twisted Alexander modules.

Our goal is to define an invariant out of $H_{1}\left(N ; \mathbb{Z}[G]\left[t^{ \pm 1}\right]\right)$. First note that by endowing $N$ with a finite cell structure we can view $C_{*}(\tilde{N}) \otimes_{\mathbb{Z}\left[\pi_{1}(N)\right]} \mathbb{Z}[G]\left[t^{ \pm 1}\right]$ as finitely generated $\mathbb{Z}\left[t^{ \pm 1}\right]$-modules. The $\mathbb{Z}\left[t^{ \pm 1}\right]$-module $H_{1}\left(N ; \mathbb{Z}[G]\left[t^{ \pm 1}\right]\right)$ is now a finitely presented and finitely related $\mathbb{Z}\left[t^{ \pm 1}\right]$-module since $\mathbb{Z}\left[t^{ \pm 1}\right]$ is Noetherian. Therefore $H_{1}\left(N ; \mathbb{Z}[G]\left[t^{ \pm 1}\right]\right)$ has a free $\mathbb{Z}\left[t^{ \pm 1}\right]$-resolution

$$
\mathbb{Z}\left[t^{ \pm 1}\right]^{r} \stackrel{S}{\rightarrow} \mathbb{Z}\left[t^{ \pm 1}\right]^{s} \rightarrow H_{1}\left(N ; \mathbb{Z}[G]\left[t^{ \pm 1}\right]\right) \rightarrow 0
$$

of finite $\mathbb{Z}\left[t^{ \pm 1}\right]$-modules. Without loss of generality we can assume that $r \geq s$.

Definition. The twisted Alexander polynomial of $(N, \alpha, \phi)$ is defined to be the order of the $\mathbb{Z}\left[t^{ \pm 1}\right]$-module $H_{1}\left(N ; \mathbb{Z}[G]\left[t^{ \pm 1}\right]\right)$, i.e. the greatest common divisor of the $s \times s$ minors of the $s \times r$-matrix $S$. It is denoted by $\Delta_{N, \phi}^{\alpha} \in \mathbb{Z}\left[t^{ \pm 1}\right]$, and it is well-defined up to units of $\mathbb{Z}\left[t^{ \pm 1}\right]$.

If $G$ is the trivial group we will drop $\alpha$ from the notation. With these conventions, $\Delta_{N, \phi} \in \mathbb{Z}\left[t^{ \pm 1}\right]$ is the ordinary 1-variable Alexander polynomial associated to $\phi$. For example, if $X(K)=S^{3} \backslash \nu K$ is the exterior of a knot $K$ and $\phi \in H^{1}(X(K) ; \mathbb{Z})$ is a generator, then $\Delta_{X(K), \phi}$ equals the ordinary Alexander polynomial $\Delta_{K}$ of a knot.

Finally, given a 3-manifold $N$ we write $H=H_{1}(N ; \mathbb{Z}) /$ torsion. Using a similar approach as above one can define the multivariable Alexander polynomial $\Delta_{N} \in \mathbb{Z}[H]$. The following theorem of Meng and Taubes [MT96] states that the multivariable Alexander polynomial of a 3-manifold $N$ corresponds to the Seiberg-Witten invariants of $N$. 
Theorem 2.5. Let $N$ be a closed 3-manifold with $b_{1}(N)>1$ and let $H=H_{1}(N ; \mathbb{Z}) /$ torsion. Then

$$
\Delta_{N}= \pm \sum_{\xi \in H^{2}(N)} S W_{N}(\xi) \cdot \frac{1}{2} f(\xi) \in \mathbb{Z}[H],
$$

where $f$ denotes the composition of Poincaré duality with the quotient map $f: H^{2}(N) \cong$ $H_{1}(N) \rightarrow H$ and, as $f(\xi)$ has even divisibility for all 3-dimensional basic classes $\xi \in$ supp $S W_{N}$, multiplication by $\frac{1}{2}$ is well-defined.

\section{Constraints from Seiberg-Witten theory}

3.1. Seiberg-Witten theory for manifolds with circle action. The essential ingredient in our approach is the fact that the Seiberg-Witten invariants of $M$ are related to the Alexander polynomial of $N$. The following theorem combines the results of Meng-Taubes [MT96] and Baldridge [Ba03, Corollaries 25 and 27] (cf. also [Ba01]), to which we refer the reader for definitions and results for Seiberg-Witten theory in this set-up:

THEOREM 3.1. Let $M$ be a 4-manifold with $b_{2}^{+}(M)>1$ admitting a free circle action with orbit space $N$. Let $e \in H^{2}(N ; \mathbb{Z})$ be the Euler class. Assume that either $e=0$ or $e$ nontorsion. Then the Seiberg-Witten invariant $S W_{M}(\kappa)$ of a class $\kappa=p^{*} \xi \in p^{*} H^{2}(N ; \mathbb{Z}) \subset$ $H^{2}(M ; \mathbb{Z})$ is given by the formula

$$
S W_{M}(\kappa)=\sum_{\xi \in\left(p^{*}\right)^{-1}(\kappa)} S W_{N}(\xi)=\sum_{\xi^{\prime}-\xi \equiv 0(e)} S W_{N}\left(\xi^{\prime}\right) \in \mathbb{Z} .
$$

Furthermore, $S W_{M}(\kappa)=0$ for any $\kappa \notin p^{*} H^{2}(N ; \mathbb{Z})$.

In the formula above, $S W_{N}(\xi)$ is the 3-dimensional SW-invariant of a class $\xi \in H^{2}(N)$, and the effect of the twisting of the $S^{1}$-fibration, measured by the class $e \in H^{2}(N)$, is to wrap up the contribution of all 3-dimensional basic classes of $N$ that have the same image in $H^{2}(M)$, i.e. that differ by a multiple of $e$. As usual, we can package the above invariants in terms of a Seiberg-Witten polynomial.

From the calculation of the Seiberg-Witten invariants of $M$ we obtain immediately the following corollary.

COROLlary 3.2. Let $M$ be a 4-manifold with $b_{2}^{+}(M)>1$ admitting a free circle action with orbit space $N$ such that the Euler class is either zero or non-torsion. Then

$$
\chi_{M}^{-}(\sigma) \geq|\sigma \cdot \sigma|+\sigma \cdot \kappa
$$

for any Seiberg-Witten basic class $\kappa \in H^{2}(M ; \mathbb{Z})$.

Proof. By (3) we only have to consider the case that $\sigma \cdot \sigma<0$. Let $\kappa$ be a basic class of $M$. Recall that this implies that $-\kappa$ is also a basic class. Let $\varphi: M \rightarrow \hat{M}$ be the orientation reversing diffeomorphism given by $\varphi(p)=p$. Given $a, b \in H^{2}(M ; \mathbb{Z})$ we have $\varphi(a)=a, \varphi(b)=b$ and $Q_{\hat{M}}(\varphi(a), \varphi(b))=-Q_{M}(a, b)$, where for sake of understanding we write explicitly the intersection forms of each manifold. 
It follows from Theorem 3.1 that $a$ is a basic class for $M$ if and only if $\varphi(a)$ is a basic class for $\hat{M}$. Applying $(3)$ to $\varphi(\sigma)$ we obtain

$$
\begin{aligned}
\chi_{M}^{-}(\sigma) & =\chi_{\hat{M}}^{-}(\varphi(\sigma)) \\
& \geq Q_{\hat{M}}(\varphi(\sigma), \varphi(\sigma))+Q_{\hat{M}}(\varphi(\sigma), \varphi(-\kappa)) \\
& =-Q_{M}(\sigma, \sigma)-Q_{M}(\sigma,-\kappa) \\
& =\left|Q_{M}(\sigma, \sigma)\right|+Q_{M}(\sigma, \kappa) .
\end{aligned}
$$

3.2. Twisted Alexander polynomials and $S W$-invariants. We are in a position now to prove our main theorem. Note that the second part is already contained in [FV08a] and [FV07].

THEOREM 3.3. Let $M$ be a 4-manifold with $b_{2}^{+}(M)>1$ admitting a free circle action with orbit space $N$. Let $e \in H^{2}(N ; \mathbb{Z})$ be the Euler class. Assume that either $e=0$ or e non-torsion. Let $\sigma \in H^{2}(M ; \mathbb{Z})$ such that $\phi=p_{*}(\sigma) \neq 0 \in H^{1}(N ; \mathbb{Z})$. Then for any epimorphism $\alpha: \pi_{1}(N) \rightarrow G$ onto a finite group we have

$$
\chi_{M}^{-}(\sigma) \geq \frac{1}{|G|}\left(\operatorname{deg}\left(\Delta_{N, \phi}^{\alpha}\right)-2 \operatorname{div} \phi_{\alpha}\right)+|\sigma \cdot \sigma| .
$$

Furthermore, if $\sigma$ is represented by a symplectic form, then $\Delta_{N, \phi}^{\alpha}$ is monic.

Proof. Let $M$ be a 4-manifold admitting a free circle action such that $b_{2}^{+}(M)>1$ and denote by $N$ its orbit space. It follows from equation (2) and the remarks which follow (2) that $b_{1}(N) \geq 2$. We will first analyze the ordinary 1-variable Alexander polynomial $\Delta_{N, \phi}$. By [FV08a] we can write this polynomial as

$$
\Delta_{N, \phi}=\left(t^{\operatorname{div} \phi}-1\right)^{2} \cdot \sum_{g \in H} a_{g} t^{\phi(g)} \in \mathbb{Z}\left[t^{ \pm 1}\right]
$$

where $H$ is the maximal free abelian quotient of $\pi_{1}(N)$ and $\Delta_{N}=\sum_{g \in H} a_{g} \cdot g \in \mathbb{Z}[H]$ is the ordinary multivariable Alexander polynomial of $N$. By Theorem 2.5 we can write

$$
\Delta_{N, \phi}= \pm\left(t^{\operatorname{div} \phi}-1\right)^{2} \sum_{\xi \in H^{2}(N)} S W_{N}(\xi) t^{\frac{1}{2} \phi \cdot \xi} .
$$

We will use now Equation (4) to write $\Delta_{N, \phi}$ in terms of the 4-dimensional Seiberg-Witten invariants of $M$. In order to do so, observe that for all classes $\xi \in H^{2}(N)$ we can write $\xi \cdot \phi=\xi \cdot p_{*}(\sigma)=p^{*}(\xi) \cdot \sigma=\kappa \cdot \sigma$ where $\kappa=p^{*}(\xi)$. Grouping together the contributions of the 3-dimensional basic classes in terms of their image in $H^{2}(M)$, and using (4) we get

$$
\begin{aligned}
\Delta_{N, \phi} & = \pm\left(t^{\operatorname{div} \phi}-1\right)^{2} \sum_{\kappa \in p^{*} H^{2}(N)} \sum_{\xi \in\left(p^{*}\right)^{-1}(\kappa)} S W_{N}(\xi) t^{\frac{1}{2} \phi \cdot \xi} \\
& = \pm\left(t^{\operatorname{div} \phi}-1\right)^{2} \sum_{\kappa \in p^{*} H^{2}(N)} S W_{M}(\kappa) t^{\frac{1}{2} \sigma \cdot \kappa} .
\end{aligned}
$$

Note that $\kappa$ is a basic class if and only if $-\kappa$ is a basic class. It now follows that

$$
\begin{aligned}
\max \{\kappa \cdot \sigma \mid \kappa \text { basic class of } M\} & =\max \left\{\kappa \cdot \sigma \mid \kappa \text { basic class of } M \text { and } \kappa \in p^{*}\left(H^{2}(N ; \mathbb{Z})\right)\right\} \\
& \geq \operatorname{deg}\left(\Delta_{N, \phi}\right)-2 \operatorname{div}(\phi) .
\end{aligned}
$$

Combining this inequality with the adjunction inequality (5) we get

$$
\chi_{M}^{-}(\sigma) \geq \operatorname{deg}\left(\Delta_{N, \phi}\right)-2 \operatorname{div}(\phi)+|\sigma \cdot \sigma| .
$$


Now assume that $\sigma$ is represented by a symplectic form $\omega$. Taubes' constraints, applied to the symplectic manifold $(M, \omega)$, assert that if $K \in H^{2}(M)$ is the canonical class, then $S W_{M}(-K)=1$. Moreover, among all basic classes $\kappa \in H^{2}(M)$, we have

$$
-K \cdot \sigma \leq \kappa \cdot \sigma
$$

with equality possible only for $\kappa=-K$. It now follows immediately from (8) that $\Delta_{N, \phi}$ is a monic polynomial.

Now let $\alpha: \pi_{1}(N) \rightarrow G$ be an epimorphism onto a finite group $G$. We denote by $\pi$ : $N_{\alpha} \rightarrow N$ the corresponding regular $G$-cover of $N$. It is well-known that $b_{1}\left(N_{\alpha}\right) \geq b_{1}(N)$. The epimorphism $\pi_{1}(M) \rightarrow \pi_{1}(N) \rightarrow G$ determines a regular $G$-cover of $M$ that we will denote (with slight abuse of notation) $\pi: M_{\alpha} \rightarrow M$. These covers are related by the commutative diagram

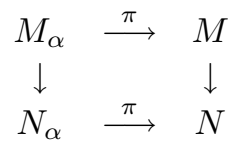

where the principal $S^{1}$-fibration $p_{\alpha}: M_{\alpha} \rightarrow N_{\alpha}$ has Euler class $e_{\alpha}=\pi^{*} e \in H^{2}\left(N_{\alpha}\right)$. Note that $e_{\alpha}=0$ whenever $e=0$, and $e_{\alpha}$ is non-torsion if and only if $e$ is non-torsion. In particular $b_{2}^{+}\left(M_{G}\right) \geq b_{2}^{+}(M)>1$.

Now let $\sigma \in H^{2}(M ; \mathbb{Z})$ and $\Sigma \subset M$ a surface of minimal complexity representing $\sigma$. Note that $\pi^{-1}(\Sigma) \subset M_{\alpha}$ is dual to $\pi^{*}(\sigma)$. We have

$$
\begin{aligned}
\chi_{M}^{-}(\sigma) & =\chi^{-}(\Sigma)=\frac{1}{|G|} \chi^{-}\left(\pi^{-1}(\Sigma)\right) \geq \frac{1}{|G|} \chi_{M_{\alpha}}^{-}\left(\pi^{*} \sigma\right), \\
\sigma \cdot \sigma & =\Sigma \cdot \Sigma=\frac{1}{|G|}\left(\pi^{-1}(\Sigma) \cdot \pi^{-1}(\Sigma)\right)=\frac{1}{|G|}\left(\pi^{*}(\sigma) \cdot \pi^{*}(\sigma)\right) .
\end{aligned}
$$

(Note that is not known whether or not $\pi^{-1}(\Sigma)$ is a surface of minimal complexity.) Applying (9) to $M_{\alpha}$ it now follows that

$$
\begin{aligned}
\chi_{M}^{-}(\sigma) & \geq \frac{1}{|G|} \chi_{M_{\alpha}}^{-}\left(\pi^{*}(\sigma)\right) \geq \frac{1}{|G|}\left(\operatorname{deg}\left(\Delta_{N_{\alpha}, \phi_{\alpha}}\right)-2 \operatorname{div}\left(\phi_{\alpha}\right)+\left|\pi^{*}(\sigma) \cdot \pi^{*}(\sigma)\right|\right) \\
& =\frac{1}{|G|}\left(\operatorname{deg}\left(\Delta_{N_{\alpha}, \phi_{\alpha}}\right)-2 \operatorname{div}\left(\phi_{\alpha}\right)\right)+|\sigma \cdot \sigma| .
\end{aligned}
$$

This, together with the relation $\Delta_{N, \phi}^{\alpha}=\Delta_{N_{\alpha}, \phi_{\alpha}}$ from [FV08a] proves the first part of the theorem.

Now assume that $\sigma$ is represented by a symplectic structure $\omega$. As $(M, \omega)$ is symplectic, $M_{\alpha}$ inherits a symplectic form $\omega_{\alpha}:=\pi^{*} \omega$ which represents $\pi^{*}(\sigma)$. Clearly $\phi_{\alpha}=p_{*}\left(\pi^{*}(\sigma)\right)$. From the above we get that $\Delta_{N_{\alpha}, \phi_{\alpha}}$ is monic. Again the equality $\Delta_{N, \phi}^{\alpha}=\Delta_{N_{\alpha}, \phi_{\alpha}}$ concludes the proof.

\section{Examples}

4.1. Applications of Theorem 1.5. Applications of Seiberg-Witten invariants to the existence of symplectic structures on 4-manifolds with a free circle action have been studied 
by many authors. In the case of trivial Euler class we refer to [Kr98, Kr99, McC01, Vi03, FV08a], in the case of non-trivial Euler class the first results were obtained by Baldridge [Ba01]. Furthermore in [FV08a] and [FV07] we give many explicit examples of 4-manifolds with a free circle action where twisted Alexander polynomials can be used to show that they do not support a symplectic structure. In this section we therefore concentrate on examples regarding the minimal complexity of surfaces in 4-manifolds with a free circle action.

Let $T$ be the 3 -torus. Recall that the 3 -torus has vanishing Thurston norm and that its multivariable Alexander polynomial is 1 . Let $x, y, z \in H_{1}(T ; \mathbb{Z})$ be a basis corresponding to the three circles of $T=S^{1} \times S^{1} \times S^{1}$ and let $C \subset T$ be a circle representing $x$. Throughout this section we denote by $\phi \in H^{1}(N ; \mathbb{Z})$ the class given by $\phi(x)=1, \phi(y)=$ $\phi(z)=0$. Pick a meridian $\mu_{C}$ and a longitude $\lambda_{C}$ for $C$ such that $\left[\mu_{C}\right]=0$ and $\left[\lambda_{C}\right]=x$ in $H_{1}(T ; \mathbb{Z})$. Next, let $K \subset S^{3}$ be an oriented knot. We denote by $\mu_{K}$ and $\lambda_{K}$ its meridian and longitude. Now, splice the two exteriors to form the 3-manifold

$$
T_{K}=(T \backslash \nu C) \cup\left(S^{3} \backslash \nu K\right)
$$

where the gluing map on the boundary 2-tori identifies $\mu_{K}$ with $\lambda_{C}$ and $\lambda_{K}$ with $\mu_{C}$.

As the surgery of Equation (12) amounts to the substitution of a solid torus with a homology solid torus, respecting the boundary maps, and as the class of $C$ is primitive, it is easy to see from the Mayer-Vietoris sequence that the inclusion maps induce isomorphisms $H_{1}(T ; \mathbb{Z}) \stackrel{\cong}{\leftrightarrows} H_{1}(T \backslash \nu C ; \mathbb{Z}) \stackrel{\cong}{\leftrightarrows} H_{1}\left(T_{K} ; \mathbb{Z}\right)$ which we use to identify these groups for the remainder of this section. We also identify $H^{1}(T ; \mathbb{Z})=H^{1}(T \backslash \nu C ; \mathbb{Z})=$ $H^{1}\left(T_{K} ; \mathbb{Z}\right)$.

Let $e=P D(z)$ and let $M_{K}(e)$ be the total space of the principal $S^{1}$-bundle over $T_{K}$ with Euler class $e$. We now specialize to the case that $K$ is the Conway knot $11_{401}$, its diagram is given in Figure 1. It is well-known that the genus of the Conway knot is 3
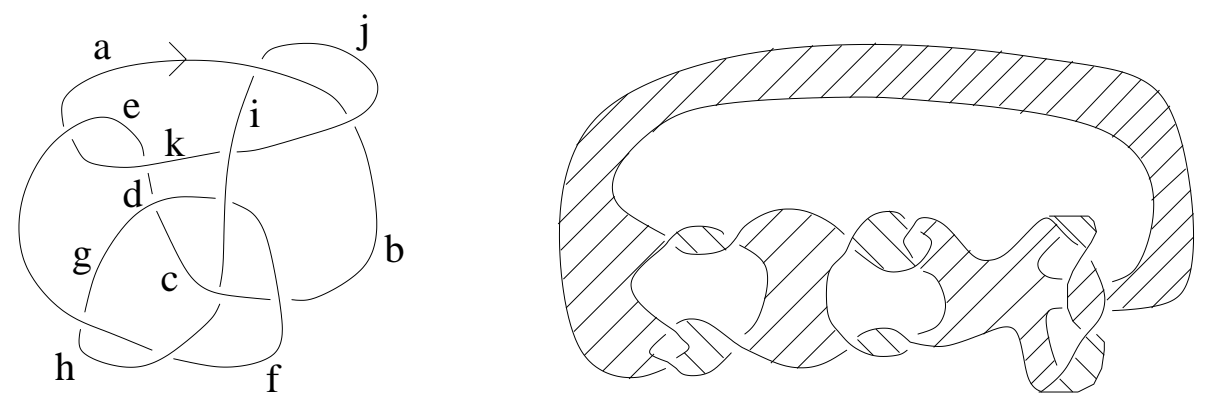

Fig. 1. The Conway knot $11_{401}$ and a Seifert surface of genus 3 (from [Ga84])

and that $\Delta_{K}=1$. Since $\partial\left(S^{3} \backslash \nu K\right)$ is an incompressible torus in $T_{K}$ it follows from the additivity of the Thurston norm that $\chi_{T_{K}}^{-}(\phi)=5+1=6$.

According to the following proposition the adjunction inequality does not determine $\chi_{M_{K}(e)}^{-}$, but for certain $\sigma$ the twisted Alexander polynomials of Theorem 1.5 detect $\chi_{M_{K}(e)}^{-}(\sigma)$. 
Proposition 4.1. Let $K \subset S^{3}$ be the Conway knot. Then

1. $S W_{M_{K}(e)}=1$,

2. For any $\sigma$ with $p_{*}(\sigma)=\phi \in H^{1}\left(T_{K} ; \mathbb{Z}\right)$ we have

$$
\chi_{M_{K}(e)}^{-}(\sigma)=\chi_{T_{K}}^{-}\left(p_{*}(\sigma)\right)+|\sigma \cdot \sigma|=6+|\sigma \cdot \sigma| .
$$

Proof. Note that if $J$ is the unknot, then $T_{J}=T$. Now we use another Alexander polynomial one knot, namely $K$ to build $T_{K}$. It is not difficult to see (using e.g. Mayer-Vietoris sequences) that $\Delta_{T_{K}}=\Delta_{T_{J}}=\Delta_{T}=1$. It follows from Theorem 2.5 and Theorem 3.1 that $S W_{M_{K}(e)}=1$.

Now let $\sigma \in H^{2}\left(M_{K}(e) ; \mathbb{Z}\right)$ with $p_{*}(\sigma)=\phi \in H^{1}\left(T_{K} ; \mathbb{Z}\right)$. First observe that by obstruction theory we can define a proper map $T \backslash \nu C \rightarrow S^{1} \times D^{2}$ which realizes the map $\pi_{1}(T \backslash \nu C) \rightarrow H_{1}(T \backslash \nu C) \rightarrow \mathbb{Z}=H_{1}\left(S^{1} \times D^{2}\right)$ given by $\phi \in H^{1}(T \backslash \nu C ; \mathbb{Z})=$ $\operatorname{Hom}\left(\pi_{1}(T \backslash \nu C), \mathbb{Z}\right)$ and which extends the diffeomorphism $\partial(T \backslash \nu C) \cong S^{1} \times \partial D^{2}$ given by identifying $\lambda_{C}$ and $\mu_{C}$ with $S^{1}$ and $\partial D^{2}$ respectively. Out of this we construct a degree one map

$$
T_{K}=(T \backslash \nu C) \cup\left(S^{3} \backslash \nu K\right) \rightarrow\left(S^{1} \times D^{2}\right) \cup\left(S^{3} \backslash \nu K\right)=N_{K}
$$

which induces an epimorphism $\pi_{1}\left(T_{K}^{3}\right) \rightarrow \pi_{1}\left(N_{K}\right)$. Given an epimorphism $\alpha: \pi_{1}\left(N_{K}\right) \rightarrow$ $G$ onto a finite group we obtain a corresponding epimorphism of $\pi_{1}\left(T_{K}\right)$ onto $G$, which we denote for simplicity by $\alpha$ as well.

We now get the following Mayer-Vietoris sequence (cf. [FK06])

$$
\begin{array}{ll}
\rightarrow H_{1}\left(S^{1} \times S^{1} ; \mathbb{Z}[G]\left[x^{ \pm 1}\right]\right) \rightarrow \begin{array}{l}
H_{1}\left(T^{3} \backslash \nu C ; \mathbb{Z}[G]\left[x^{ \pm 1}\right]\right) \\
H_{1}\left(S^{3} \backslash \nu K ; \mathbb{Z}[G]\left[x^{ \pm 1}\right]\right)
\end{array} \rightarrow H_{1}\left(T_{K} ; \mathbb{Z}[G]\left[x^{ \pm 1}\right]\right) \rightarrow \\
\rightarrow H_{0}\left(S^{1} \times S^{1} ; \mathbb{Z}[G]\left[x^{ \pm 1}\right]\right) \rightarrow \begin{array}{l}
H_{0}\left(T^{3} \backslash \nu C ; \mathbb{Z}[G]\left[x^{ \pm 1}\right]\right) \\
H_{0}\left(S^{3} \backslash \nu K ; \mathbb{Z}[G]\left[x^{ \pm 1}\right]\right)
\end{array} \rightarrow H_{0}\left(T_{K} ; \mathbb{Z}[G]\left[x^{ \pm 1}\right]\right) \rightarrow
\end{array}
$$

First note that the maps

$$
\begin{aligned}
& \phi \times \alpha: \pi_{1}\left(S^{1} \times S^{1}\right) \rightarrow \mathbb{Z} \times G \\
& \phi \times \alpha: \pi_{1}\left(T^{3} \backslash \nu C\right) \rightarrow \mathbb{Z} \times G
\end{aligned}
$$

both factor through $\phi$. In particular we get a commutative diagram

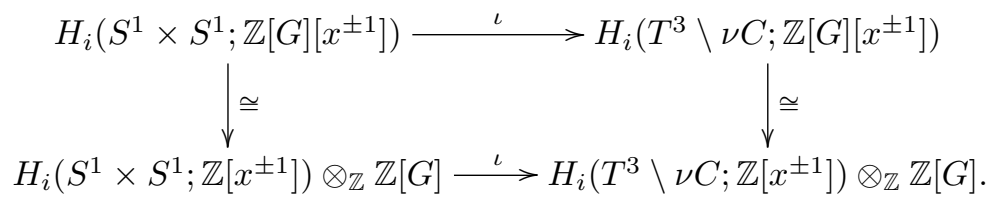

It is now easy to see that the bottom map is an isomorphism for $i=0$. Furthermore $H_{1}\left(S^{1} \times S^{1} ; \mathbb{Z}\left[x^{ \pm 1}\right]\right)$ is given by $\mathbb{Z} c$ where $c$ is a curve representing the commutator $[y, z]$. On the other hand $H_{1}\left(T^{3} \backslash \nu C ; \mathbb{Z}\left[x^{ \pm 1}\right]\right)$ is given by $\mathbb{Z} y \oplus \mathbb{Z} z$. We see that the inclusion induced map $H_{1}\left(S^{1} \times S^{1} ; \mathbb{Z}\left[x^{ \pm 1}\right]\right) \rightarrow H_{1}\left(T^{3} \backslash \nu C ; \mathbb{Z}\left[x^{ \pm 1}\right]\right)$ is trivial. It follows from this discussion that the above Mayer-Vietoris sequence descends to a sequence of the form:

$$
0 \rightarrow \mathbb{Z}^{|G|} \oplus \mathbb{Z}^{|G|} \oplus H_{1}\left(S^{3} \backslash \nu K ; \mathbb{Z}[G]\left[x^{ \pm 1}\right]\right) \rightarrow H_{1}\left(T_{K} ; \mathbb{Z}[G]\left[x^{ \pm 1}\right]\right) \rightarrow 0 .
$$


It follows that

$$
\begin{aligned}
\operatorname{deg}\left(\Delta_{T_{K}, \phi}^{\alpha}\right) & =\operatorname{rank}_{\mathbb{Z}}\left(H_{1}\left(T_{K} ; \mathbb{Z}[G]\left[x^{ \pm 1}\right]\right)\right)=\operatorname{rank}_{\mathbb{Z}}\left(H_{1}\left(S^{3} \backslash \nu K ; \mathbb{Z}[G]\left[x^{ \pm 1}\right]\right)\right)+2|G| \\
& =\operatorname{deg}\left(\Delta_{K, \phi}^{\alpha}\right)+2|G| .
\end{aligned}
$$

We now pick a particular epimorphism $\alpha: \pi_{1}\left(N_{K}\right) \rightarrow G$. The fundamental group $\pi_{1}\left(N_{K}\right)$ is generated by the meridians $a, b, \ldots, k$ of the segments in the knot diagram of Figure 1. The relations are

$$
\begin{aligned}
& a=j b j^{-1}, \quad b=f c f^{-1}, \quad c=g^{-1} d g, \quad d=k^{-1} e k, \\
& e=h^{-1} f h, \quad f=i g i^{-1}, \quad g=e^{-1} h e, \quad h=c^{-1} i c, \\
& i=a j a^{-1}, \quad j=i k i^{-1}, \quad k=e^{-1} a e, \quad a^{-1}=j f g^{-1} k^{-1} h^{-1} i e^{-1} c^{-1} a i e^{-1} .
\end{aligned}
$$

Using the program KnotTwister [Fr07] we found the homomorphism $\varphi: \pi_{1}\left(N_{K}\right) \rightarrow A_{5}$ given by

$$
\begin{aligned}
& a \mapsto(142), \quad b \mapsto(451), \quad c \mapsto(451), \quad d \mapsto(453), \\
& e \mapsto(453), \quad f \mapsto(351), \quad g \mapsto(351), \quad h \mapsto(431), \\
& i \mapsto(351), \quad j \mapsto(352), \quad k \mapsto(321),
\end{aligned}
$$

where we use cycle notation. Using KnotTwister we compute that the degree of $\Delta_{K, \phi}^{\alpha}$ computed as an element in $\mathbb{F}_{53}\left[t^{ \pm 1}\right]$ equals 209. It follows from [FV08b, Proposition 6.1] that the degree of $\Delta_{K, \phi}^{\alpha}$ computed as an element in $\mathbb{Z}\left[t^{ \pm 1}\right]$ equals at least 209. We also have $\operatorname{div}\left(\phi_{\alpha}\right)=1$. It now follows from Theorem 1.5 that

$$
\begin{aligned}
\chi_{M_{K}(e)}^{-}(\sigma) & \geq \frac{1}{\left|A_{5}\right|}\left(\operatorname{deg}\left(\Delta_{T_{K}, \phi}^{\alpha}\right)-2 \operatorname{div} \phi_{\alpha}\right)+|\sigma \cdot \sigma| \\
& =\frac{1}{60}\left(\operatorname{deg}\left(\Delta_{K, \phi}^{\alpha}\right)+2 \cdot 60-2\right)+|\sigma \cdot \sigma| \\
& \geq \frac{1}{60}(209+2 \cdot 60-2)+|\sigma \cdot \sigma|=\frac{327}{60}+|\sigma \cdot \sigma| .
\end{aligned}
$$

Lemma 2.2 asserts that $\chi_{M_{K}(e)}^{-}(\sigma) \leq 6+|\sigma \cdot \sigma|$, as $\chi_{T_{K}}(\phi)$ equals 6 . The claim now follows from Lemma 2.2 and the fact that $\chi_{M_{K}(e)}^{-}$is necessarily an integer.

4.2. The limitations of Theorem 1.5. The following lemma says that in many cases all twisted Alexander polynomials will be zero and therefore Theorem 1.5 will not be able to give any information on the minimal complexity of surfaces.

LEMmA 4.2. Let $N$ be a 3-manifold and $\phi \in H^{1}(N ; \mathbb{Z})$ such that $\Delta_{N, \phi}=0$. Then $\Delta_{N, \phi}^{\alpha}=$ 0 for all epimorphisms $\alpha: \pi_{1}(N) \rightarrow G$ to a finite group $G$.

As an example, note that for any $N$ which is the direct sum of $N_{1}, N_{2}$ with $b_{1}\left(N_{i}\right) \geq 1$ we have $\Delta_{N, \phi}=0$ for any $\phi \in H^{1}(N ; \mathbb{Z})$. This can be seen using a straightforward MayerVietoris argument. Another example is given by any $N$ which is the 0-framed surgery on a boundary link.

Proof. First note that for an epimorphism $\beta: \pi_{1}(N) \rightarrow H$ to a finite group $H$ we have $\Delta_{N, \phi}^{\beta}=0$ if and only if

$$
\operatorname{dim}_{\mathbb{Q}}\left(H_{1}\left(N ; \mathbb{Q}[H]\left[t^{ \pm 1}\right]\right)\right)=\infty
$$


By Maschke's theorem (cf. e.g. [Ro96, p. 216]) we have a direct sum decomposition of $\mathbb{Q}[G]$-modules

$$
\mathbb{Q}[G]=\mathbb{Q} \oplus V,
$$

where $\mathbb{Q}$ is the $\mathbb{Q}[G]$-module with the trivial $G$-action. It follows that

$$
\left(H _ { 1 } ( N ; \mathbb { Q } [ G ] [ t ^ { \pm 1 } ] ) \cong \left(H _ { 1 } ( N ; \mathbb { Q } [ t ^ { \pm 1 } ] ) \oplus \left(H_{1}\left(N ; V \otimes_{\mathbb{Q}} \mathbb{Q}\left[t^{ \pm 1}\right]\right) .\right.\right.\right.
$$

The lemma now follows immediately from the above observation applied to $H=e$ and $H=G$.

Acknowledgments. S. Friedl was supported by a CRM-ISM Fellowship and by CIRGET. S. Vidussi was partially supported by a University of California Regents' Faculty Fellowships.

The first author would like to thank the organizers of the Postnikov memorial conference in Będlewo, Poland for the relaxed atmosphere which allowed for many interesting conversations. We also would like to thank the referee for various helpful comments.

\section{References}

[Ba01] S. Baldridge, Seiberg-Witten invariants of 4-manifolds with free circle actions, Commun. Contemp. Math. 3 (2001), 341-353.

[Ba03] S. Baldridge, Seiberg-Witten invariants, orbifolds, and circle actions, Trans. Amer. Math. Soc. 355 (2003), 1669-1697.

[Bou88] A. Bouyakoub, Sur les fibrés principaux de dimension 4, en tores, munis de structures symplectiques invariantes et leurs structures complexes, C. R. Acad. Sci. Paris Sér. I Math. 306 (1988), 417-420.

[Bow07] J. Bowden, The topology of symplectic circle bundles, Trans. Amer. Math. Soc., to appear.

[Ch03] J. Cha, Fibred knots and twisted Alexander invariants, Trans. Amer. Math. Soc. 355 (2003), 4187-4200.

[FGM91] M. Fernández, A. Gray and J. W. Morgan, Compact symplectic manifolds with free circle actions, and Massey products, Michigan Math. J. 38 (1991), 271-283.

[Fr07] S. Friedl, KnotTwister, http://www.labmath.uqam.ca/ ${ }^{\sim}$ friedl/index.html (2007).

[FK06] S. Friedl and T. Kim, The Thurston norm, fibered manifolds and twisted Alexander polynomials, Topology 45 (2006), 929-953.

[FV08a] S. Friedl and S. Vidussi, Twisted Alexander polynomials and symplectic structures, Amer. J. Math. 130 (2008), 455-484.

[FV08b] S. Friedl and S. Vidussi, Symplectic $\mathbf{S}^{1} \times \mathbf{N}^{3}$, surface subgroup separability, and totally degenerate Thurston norm, J. Amer. Math. Soc. 21 (2008), 597-610.

[FV07] S. Friedl and S. Vidussi, Symplectic 4-manifolds with a free circle action, preprint, 2007.

[Ga83] D. Gabai, Foliations and the topology of 3-manifolds, J. Differential Geom. 18 (1983), 445-503.

[Ga84] D. Gabai, Foliations and genera of links, Topology 23 (1984), 381-394.

[GKM05] H. Goda, T. Kitano and T. Morifuji, Reidemeister torsion, twisted Alexander polynomial and fibred knots, Comment. Math. Helv. 80 (2005), 51-61. 
[KL99] P. Kirk and C. Livingston, Twisted Alexander invariants, Reidemeister torsion and Casson-Gordon invariants, Topology 38 (1999), 635-661.

[Ki08] T. Kitayama, Normalization of twisted Alexander invariants, preprint, 2008.

[Kr98] P. Kronheimer, Embedded surfaces and gauge theory in three and four dimensions, in: Surveys in Differential Geometry, Vol. III (Cambridge, MA, 1996), Int. Press, Boston, MA 1998, 243-298.

[Kr99] P. Kronheimer, Minimal genus in $S^{1} \times M^{3}$, Invent. Math. 135 (1999), 45-61.

[Li01] X. S. Lin, Representations of knot groups and twisted Alexander polynomials, Acta Math. Sin. (Engl. Ser.) 17 (2001), 361-380.

[McC01] J. McCarthy, On the asphericity of a symplectic $M^{3} \times S^{1}$, Proc. Amer. Math. Soc. 129 (2001), 257-264.

[McM02] C. T. McMullen, The Alexander polynomial of a 3-manifold and the Thurston norm on cohomology, Ann. Sci. École Norm. Sup. (4) 35 (2002), 153-171.

[MT96] G. Meng and C. H. Taubes, $S W=$ Milnor torsion, Math. Res. Lett. 3 (1996), 661-674.

[Ro96] D. Robinson, A Course in the Theory of Groups, Graduate Texts in Mathematics 80, Springer Verlag, 1996.

[Ta94] C. H. Taubes, The Seiberg-Witten invariants and symplectic forms, Math. Res. Lett. 1 (1994), 809-822.

[Ta95] C. H. Taubes, More constraints on symplectic forms from Seiberg-Witten invariants, Math. Res. Lett. 2 (1995), 9-13.

[Th76] W. P. Thurston, Some simple examples of symplectic manifolds, Proc. Amer. Math. Soc. 55 (1976), 467-468.

[Th86] W. P. Thurston, A norm for the homology of 3-manifolds, Mem. Amer. Math. Soc. 339 (1986), 99-130.

[Tu02] V. Turaev, A homological estimate for the Thurston norm, preprint (2002), arXiv:math. GT/0207267.

[Vi03] S. Vidussi, Norms on the cohomology of a 3-manifold and $S W$ theory, Pacific J. Math. 208 (2003), 169-186.

[Wa94] M. Wada, Twisted Alexander polynomial for finitely presentable groups, Topology 33 (1994), 241-256. 
